\title{
Therapeutic enteroscopy using a new single-balloon enteroscope: a case series
}

Authors

Institution
Tom G. Moreels, Nathalie Kouinche Madenko, Alaa Taha, Hubert Piessevaux, Pierre H. Deprez

Cliniques universitaires Saint-Luc, Hépato-Gastroentérologie, Brussels, Belgium

\section{Bibliography}

DOI http://dx.doi.org/

10.1055/s-0042-111205

Published online: 10.8.2016

Endoscopy International Open 2016; 04: E918-E921

(c) Georg Thieme Verlag KG

Stuttgart · New York

E-ISSN 2196-9736

\section{Corresponding author} Tom G Moreels, MD PhD

Cliniques universitaires SaintLuc

Hépato-Gastroentérologie

Av. Hippocrate 10

B-1200 Brussels

BELGIUM

Phone: +32 27642892

Fax: +32 27649171

tom.moreels@uclouvain.be
Background and study aims: Balloon-assisted enteroscopy allows therapeutic intervention in the small bowel, and even of the biliopancreatic system in patients with altered anatomy. However, the conventional single-balloon enteroscope (SBE) has limited therapeutic use because of its small-caliber working channel and the lack of an additional water jet channel. The new single-balloon enteroscope prototype XSIF-180JY has been developed to overcome these problems. We present experience with use of the new SBE prototype during 14 therapeutic endoscopy procedures, which illustrates its advantages.

Patients and methods: During a 2-month period, 16 SBE procedures were performed (2 antegrade, 2 retrograde and 12 ERCP procedures) using the XSIF-180JY prototype, 14 of which were done with therapeutic intent.

Results: The XSIF-180JY SBE allowed deep enteroscopy with balloon dilation and multiple intes-

\section{Introduction}

$\nabla$

Single-balloon enteroscopy (SBE) has been commercially available since 2007 [1,2]. It allows deep enteroscopy and even endoscopic retrograde cholangiopancreatography (ERCP) in patients with altered anatomy [3]. However, due to the slim caliber $(9.2 \mathrm{~mm})$ of the commercially available SIF-Q180 SBE, its working channel is limited to $2.8 \mathrm{~mm}$. This may render the introduction of accessory catheters difficult with too much friction, especially when the enteroscope is in a torqued position [4]. Moreover, the SIF-Q180 SBE has no additional water jet channel. Therefore, the working channel must be cleared in order to use it as a water jet channel for flushing. Recently, a shorter $(152 \mathrm{~cm})$ therapeutic SIF-Y0004 SBE has been developed with a 3.2-mm working channel and an additional water jet channel, permitting ERCP in patients with short-limb Roux-en-Y altered small bowel anatomy [5]. These adaptations tinal polypectomies. Moreover, 14 ERCP procedures were successfully performed in 12 patients with Roux-en-Y altered anatomy. Sphincterotomy, balloon dilation, stone extraction and $7 \mathrm{Fr}$ plastic stent placement were performed through the 3.2-mm working channel. The additional water jet was useful for flushing away stone fragments from the intrahepatic bile ducts and the retrieval basket and for flushing away blood from a bleeding sphincterotomy. No complications related to the enteroscope were encountered.

Conclusions: The new therapeutic XSIF-180JY SBE permitted therapeutic enteroscopy and ERCP through its 3.2-mm working channel and the additional water jet channel proved useful in flushing away biliary stones and blood without the need to clear the working channel. This newly developed SBE has the advantage of a larger working channel and an additional water jet, improving therapeutic enteroscopy.

have now been built into the 200-cm XSIF-180JY SBE prototype with a $3.2-\mathrm{mm}$ working channel and an additional water jet channel without increasing the enteroscope's outer diameter ( $\bullet$ Fig. 1). These characteristics may improve the therapeutic use of SBE, also in patients with long-limb Roux-en-Y-altered anatomy.

\section{Patients and methods}

$\nabla$

We evaluated the XSIF-180JY SBE during a 2month period (January and February 2016). Data on all procedures were retrospectively collected. In total 16 SBE procedures were performed on 14 patients during the study period. In 12 patients, 14 procedures were performed with therapeutic intent and were used for further analysis. All patients signed the informed consent to undergo balloon-assisted enteroscopy. The XSIF-180JY SBE was used with the conventional commercially 


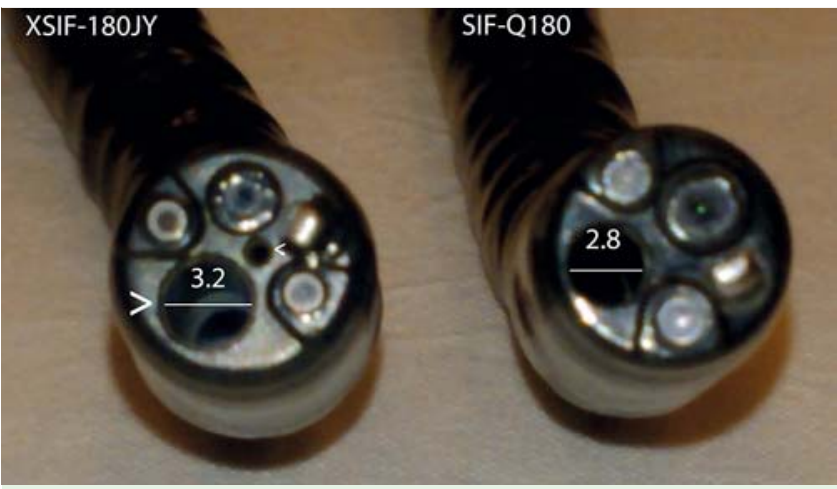

Fig. 1 Differences between the commercially available SIF-Q180 SBE and the XSIF-180JY SBE prototype: XSIF-180JY has a larger working channel (large arrow) caliber of $3.2 \mathrm{~mm}$ and an additional water jet channel (small arrow).

available ST-SB1 overtube and the OBCU balloon control unit. All procedures were performed in supine position under general anesthesia with endotracheal intubation and with $\mathrm{CO}_{2}$-insufflation. Fluoroscopy was available when indicated. We analyzed the indications and therapeutic success of SBE using XSIF-180JY and evaluated the possible advantages of a larger working channel and additional water jet channel. Descriptive statistics were performed using Excel 2013 for Windows.

The following accessory catheters were used: cannulation catheter (PR-Y0001), sphincterotome (KD-Y0005), extraction balloon (B-Y0003), retrieval basket (FG-Y0003) and stent pusher (MAJY0025-1) were Olympus prototypes (Olympus Medical Systems Corp., Japan); guidewire (0.025 inch $450 \mathrm{~cm}$ Jagwire Guidewire ST), dilation balloon (8-10 mm CRE Microvasive), polypectomy snare (Captivator), biopsy forceps (Radial Jaw 4) were from Boston Scientific (Ireland); plastic biliary stent (7 Fr), hemostatic clip (Instinct Endoscopic Hemoclip) were from Cook Medical (Ireland), injection needle was from Prince Medical (France).

\section{Results}

The male/female ratio was $3 / 9(25 / 75 \%)$ with a mean age of 50 years (range 8 -87). As shown in $\bullet$ Table 1 , the main indication was cholangitis in 10 patients with Roux-en-Y-altered anatomy.
In total, 12 ERCP procedures were successfully performed in 8 patients with short-limb Roux-en-Y and biliary anastomosis (biliary surgery with bilioenteric anastomosis, Roux-en-Y liver transplantation and Roux-en-Y Whipple resection) or intact papilla (Roux-en-Y gastrectomy) and in 2 patients with long-limb Rouxen-Y and intact papilla (RouX-en-Y gastric bypass). In all 10 patients, the biliary system was reached and ERCP was successful. Cholangitis due to stenosis of the biliary anastomosis or to bile duct stones was the indication for performing ERCP in patients with Roux-en-Y-altered anatomy. In case of a stenotic biliary anastomosis, cannulation was performed using the PR-Y0001 cannulation catheter and the 0.025 -inch guidewire was introduced into the biliary system. Then the cannulation catheter was replaced over the guidewire with an 8- to $10-\mathrm{mm}$ dilation balloon to perform progressive dilation of the anastomosis under fluoroscopic and endoscopic control. The balloon was filled with contrast to evaluate the complete dilation of the stenosis. In case of a native papilla, cannulation was performed using the PRY0001 cannulation catheter with the 0.025-inch guidewire. Next, the cannulation catheter was replaced by the KD-Y0005 sphincterotome over the guidewire and sphincterotomy was performed. When indicated, stone extraction was performed using the B-Y0003 extraction balloon. In 2 patients, the use of the FGY0003 retrieval basket was necessary to clear the common and intrahepatic bile ducts from multiple stones. In 4 patients, 1 or 2 $7 \mathrm{Fr}$ plastic stents were inserted over the guidewire using the MAJ-Y0025-1 stent pusher to improve biliary drainage after balloon dilation of the biliary anastomosis or after sphincterotomy. One of the gastric bypass patients referred for SBE ERCP because of biliary obstruction appeared to have an ampulloma. Sphincterotomy and plastic stent insertion was performed and biopsies of the papilla confirmed the diagnosis. The endoscopic or surgical resection of the ampulloma remains to be discussed.

Introduction and use of accessory catheters via the $3.2-\mathrm{mm}$ working channel was considered feasible without excessive friction, even with the tip of the enteroscope in the retrovision position ( $\bullet$ Fig. 2). In 2 patients with bilioenteric anastomosis complicated by intrahepatic biliary stones, the anastomosis was dilated up to $10 \mathrm{~mm}$, allowing the water jet channel to flush the biliary stones into the intestinal limb without the need for an extraction balloon or basket. When using a stone retrieval basket, stones were cleared from the basket by using the water jet channel ( Fig. 3). Plastic 7 Fr stents were easily introduced when in-

Table 1 Case series of therapeutic enteroscopy using the XSIF-180JY SBE.

\begin{tabular}{|c|c|c|c|c|}
\hline Patient/Age & Indication & Anatomy & Intervention & SBE procedure \\
\hline Female $34 y$ & Peutz-Jeghers syndrome & Normal & Multiple intestinal polypectomies & Antegrade SBE \\
\hline Female 43y & ileal Crohn's disease & Ileocecal resection & Balloon dilation ileal stenosis & Retrograde SBE \\
\hline \multirow[t]{2}{*}{ Male 23y } & Cholangitis & Roux-en-Y biliary anastomosis & Balloon dilation anastomosis & SBE ERCP \\
\hline & Cholangitis & Roux-en-Y biliary anastomosis & Balloon dilation anastomosis/2 plastic stents & SBE ERCP \\
\hline Female $63 y$ & Cholangitis & Roux-en-Y biliary anastomosis & Balloon dilation anastomosis & SBE ERCP \\
\hline Female $61 \mathrm{y}$ & Cholangitis & Roux-en-Y biliary anastomosis & Balloon dilation anastomosis & SBE ERCP \\
\hline Female $8 y$ & Cholangitis & Roux-en-Y biliary anastomosis & Balloon dilation anastomosis/stone extraction & SBE ERCP \\
\hline Male $67 y$ & Cholangitis & Roux-en-Y Whipple resection & $\begin{array}{l}\text { Balloon dilation anastomosis/stone extraction/ } \\
\text { plastic stent }\end{array}$ & SBE ERCP \\
\hline Male 61y & Cholangitis & Roux-en-Y gastrectomy & sphincterotomy/plastic stent & SBE ERCP \\
\hline Female $83 y$ & Cholangitis & Roux-en-Ygastrectomy & Sphincterotomy/stone extraction/plastic stent & SBE ERCP \\
\hline \multirow[t]{2}{*}{ Female $87 y$} & Cholangitis & Roux-en-Ygastrectomy & Sphincterotomy & SBE ERCP \\
\hline & Bleeding sphincterotomy & Roux-en-Ygastrectomy & Hemostasis & SBE ERCP \\
\hline Female $41 y$ & Cholangitis & Roux-en-Y gastric bypass & Sphincterotomy/stone extraction & SBE ERCP \\
\hline Female $62 y$ & Cholangitis & Roux-en-Y gastric bypass & Sphincterotomy/plastic stent & SBE ERCP \\
\hline
\end{tabular}




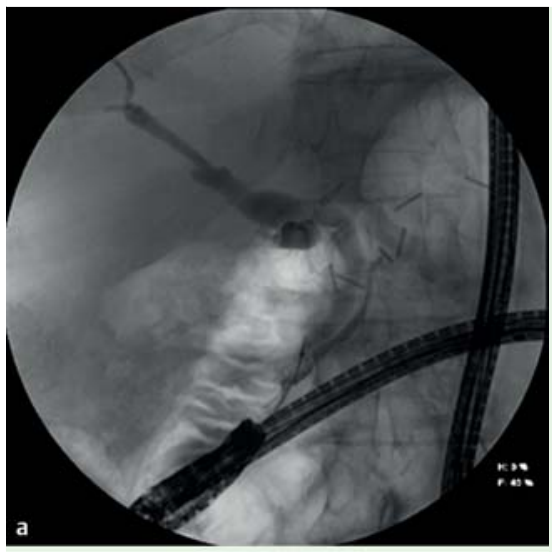

Fig. 2 Cholangiography with the XSIF-180JY in the retroflex position in a patient with shorttype Roux-en-Y total gastrectomy (a) and the long-type Roux-en-Y gastric bypass (b). Thanks to the larger working channel, introduction of the cannulation catheter (a) or the extraction balloon (b) was feasible without excessive friction.
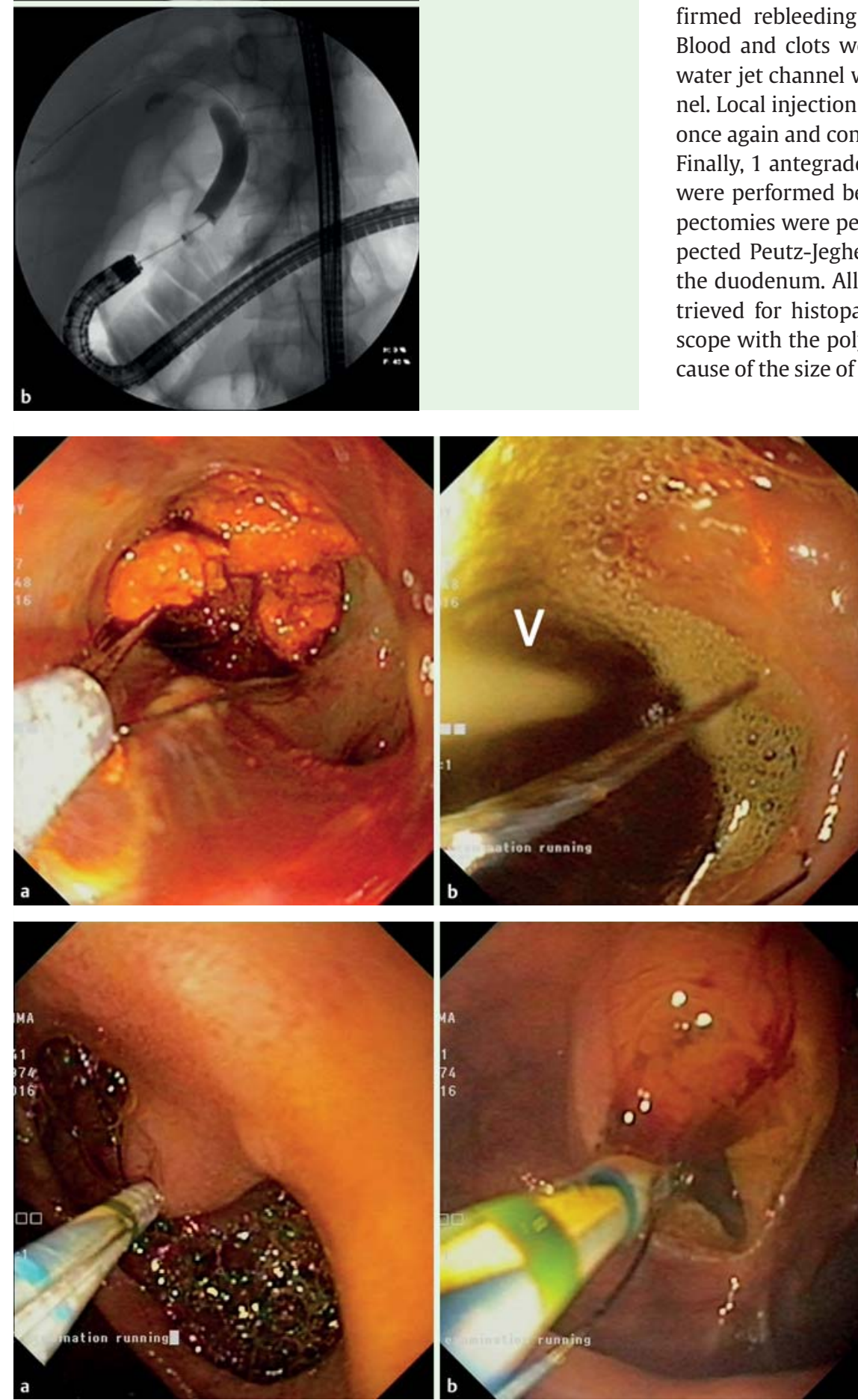

dicated without excessive friction. In addition, when using a forward-looking SBE without elevator handle, the direction of the sphincterotome may vary and rotation of the sphincterotome often is necessary to orient the cutting wire. That procedure was feasible with the sphincterotome inside the 3.2-mm working channel ( $\bullet$ Fig. 4$)$.

One adverse event (AE) was encountered during ERCP in an 87year-old patient with Roux-en-Y total gastrectomy and cholangitis due to common bile duct stones. Over-the-wire sphincterotomy was performed but resulted in spurting arterial bleeding. Initial hemostasis was obtained after local submucosal injection of 4 cc of 1/100,000 epinephrine. However, 2 days later, the patient developed melena and the hemoglobin level dropped $2 \mathrm{~g} /$ dL. Urgent SBE was performed using XSIF-180JY, which confirmed rebleeding at the level of the recent sphincterotomy. Blood and clots were easily flushed away using the additional water jet channel with the needle catheter in the working channel. Local injection of 4 cc 1/100,000 epinephrine was performed once again and controlled the bleeding.

Finally, 1 antegrade and 1 retrograde therapeutic SBE procedure were performed because of intestinal pathology. Multiple polypectomies were performed in the jejunum in a patient with suspected Peutz-Jeghers syndrome, from the distal jejunum up to the duodenum. All polyps were endoscopically resected and retrieved for histopathologic analysis by removal of the enteroscope with the polyp, leaving the inflated overtube in place. Because of the size of the polyps ( $>20 \mathrm{~mm}$ ), retrieval was performed

Fig. 3 Ilustration of the usefulness of the additional water jet channel: clearance of multiple biliary stones (a) from the retrieval basket by means of the water jet (arrow) (b).

Fig.4 Ilustration of the rotatability of the sphincterotome catheter (a) in the clockwise direction inside the XSIF-180jY working channel in order to correctly orient the direction of the cutting wire at the level of the papilla (distal approach) (b). 
through the overtube. After each polypectomy, the enteroscope was reintroduced into the overtube to perform the next polypectomy. When deemed indicated to prevent bleeding or perforation, rotatable hemoclips were placed in order to close the mucosal defect after intestinal polypectomy. Insertion and rotation of the hemoclip catheter was feasible using the XSIF-180JY SBE. In 1 patient with Crohn's disease, retrograde SBE was performed successfully in order to dilate a 2 -cm ileal stricture.

\section{Discussion}

Balloon-assisted enteroscopy has become the gold standard method for performing therapeutic endoscopy in the small bowel. It allows nearly all conventional therapeutic interventions at the level of the small bowel, and also ERCP in patients with altered anatomy [3]. However, currently available single- and double-balloon enteroscopes with a working length of $200 \mathrm{~cm}$ have a working channel of only $2.2 \mathrm{~mm}$ (EN-450P5/20 DBE) or $2.8 \mathrm{~mm}$ (EN-450T5 DBE, SIF-Q180 SBE), rendering the use of accessory material sometimes difficult or even impossible [4]. In order to improve its therapeutic use, Olympus developed the XSIF-180JY single-balloon enteroscope with a working channel of $3.2 \mathrm{~mm}$ and an additional water jet channel without increasing the enteroscope's outer diameter of $9.2 \mathrm{~mm}$. Therefore, this new enteroscope can be used with the conventional ST-SB1 overtube and the OBCU balloon control unit. The larger working channel limits the friction while introducing accessory catheters and facilitates their use [4]. The water jet channel allows rinsing of the intestinal lumen, or even the biliary system with an accessory catheter inside the working channel for therapeutic interventions.

We evaluated this newly developed SBE during a 2-month period. We present 14 therapeutic enteroscopy procedures using the new XSIF-180JY SBE prototype, illustrating its advantages. All procedures were successful and no AEs were encountered, apart from one incident of sphincterotomy bleeding requiring endoscopic treatment. All the procedures were done with therapeutic intent, ERCP in patients with altered anatomy being the most frequent indication (86\%). With the XSIF-180JY SBE, it was possible to reach the intact papilla or the biliary anastomosis in all cases, even in patients with long-limb Roux-en-Y reconstruction.

Using the Olympus cannulation catheter (PR-Y0001), cholangiography was obtained followed by the indicated therapeutic biliary intervention (balloon dilation of the biliary anastomosis, sphincterotomy, stone extraction, plastic stent placement, hemostasis). The 3.2-mm working channel allowed swift introduction of all catheters and $7 \mathrm{Fr}$ stents, even with the tip of the enteroscope in the retroflex position. With the SIF-Q180 model, introduction of accessory catheters is not possible in the retroflex position, rendering their well-controlled and precise use sometimes difficult or even impossible. Moreover, rotation of the sphincterotome or the hemostatic clip was feasible even when the enteroscope was in a torqued position.

In 1 patient with suspected Peutz-Jeghers syndrome, multiple large size polyps were resected from the jejunum and recuper- ated for histopathologic analysis. By leaving the overtube in place, the enteroscope with the polyp was retrieved after each polypectomy, as previously described [6]. The XSIF-180JY SBE was easily removed from and reintroduced in the overtube in order to perform multiple intestinal polypectomies with recovery of the large polyps for histopathologic analysis. Retrograde enteroscopy also was feasible using the XSIF-180JY SBE, as shown in a patient with stricturing ileal Crohn's disease. Finally, the additional water jet channel proved useful for removal of biliary sludge and stones form the biliary tract or from the stone retrieval basket. Moreover, arterial bleeding after sphincterotomy in a patient with Roux-en-Y total gastrectomy was quickly controlled by flushing away the blood through the water jet channel and immediately injecting epinephrine through the $3.2-\mathrm{mm}$ working channel. That is not possible with the conventional SIF-Q180 $\mathrm{SBE}$, which has only a $2.8-\mathrm{mm}$ working channel and no additional water jet channel.

\section{Conclusions \\ $\nabla$}

In conclusion, the adaptations of the XSIF-180JY prototype improved the therapeutic use of SBE. The larger working channel allowed easy access to all accessory catheters, even when the enteroscope was in the retroflex position. The additional water jet channel proved useful for endoscopic treatment of biliary stones/sludge and an incident of arterial sphincterotomy bleeding. Moreover, these adaptations were made while maintaining the enteroscope's outer diameter. Therefore, the XSIF-180JY SBE can be used with the commercially available overtube and balloon control unit.

\section{Competing interests: None}

\section{Acknowledgements \\ $\nabla$}

Olympus Belgium allowed us to evaluate the newly developed XSIF-180JY SBE prototype and provided us with free accessory ERCP catheters.

\section{References}

1 Hartmann D, Eickhoff A, Tamm $R$ et al. Balloon-assisted enteroscopy using a single-balloon technique. Endoscopy 2007; 39: E276

2 Moreels TG. History of endoscopic devices for the exploration of the small bowel. Acta Gastroenterol Belg 2009; 72: 335-337

3 Moreels TG. Altered anatomy: enteroscopy and ERCP procedure. Best Pract Res Clin Gastroenterol 2012; 26: 347-357

4 Kawashima H, Nakamura M, Ohno E et al. Impact of instrument channel diameter on therapeutic endoscopic retrograde cholangiography using balloon-assisted enteroscopy. Dig Endosc 2014; 26: 127-129

5 Yamauchi H, Kida M, Okuwaki K et al. Passive-bending, short-type single-balloon enteroscope for endoscopic retrograde cholangiopancreatography in Roux-en-Y anastomosis patients. World J Gastroenterol 2015; 21: $1546-1553$

6 Kröner PT, Sancar A, Fry LC et al. Endoscopic mucosal resection of jejunal polyps using double-balloon enteroscopy. GE J Port Gastroenterol 2015; 22: 137-142 